\title{
L'agrobiodiversité végétale au sein des paysages forestiers utilisés par les communautés Baka et Konabembé au Sud-Est Cameroun
}

Caractérisation et potentiel pour la sécurité alimentaire des ménages

Plant agrobiodiversity in the forest landscapes used by the Baka and Konabembe communities in South-East Cameroon: characterization and potential for household food security

Évariste Fongnzossie, Thomas Mbang Nkongo, Didier Dibong Siegfried et Marlene Ngansop

\section{OpenEdition}

\section{Journals}

Édition électronique

URL : http://journals.openedition.org/ethnoecologie/3413

DOI : 10.4000/ethnoecologie.3413

ISSN : 2267-2419

Éditeur

Laboratoire Eco-anthropologie et Ethnobiologie

Référence électronique

Évariste Fongnzossie, Thomas Mbang Nkongo, Didier Dibong Siegfried et Marlene Ngansop, «

L'agrobiodiversité végétale au sein des paysages forestiers utilisés par les communautés Baka et

Konabembé au Sud-Est Cameroun », Revue d'ethnoécologie [En ligne], 13 | 2018, mis en ligne le 25 juin

2018, consulté le 20 avril 2019. URL : http://journals.openedition.org/ethnoecologie/3413 ; DOI :

10.4000/ethnoecologie.3413

Ce document a été généré automatiquement le 20 avril 2019

Revue d'ethnoécologie est mis à disposition selon les termes de la licence Creative Commons Attribution - Pas d'Utilisation Commerciale - Pas de Modification 4.0 International. 


\section{L'agrobiodiversité végétale au sein des paysages forestiers utilisés par les communautés Baka et Konabembé au Sud-Est Cameroun}

Caractérisation et potentiel pour la sécurité alimentaire des ménages

Plant agrobiodiversity in the forest landscapes used by the Baka and Konabembe communities in South-East Cameroon: characterization and potential for household food security

Évariste Fongnzossie, Thomas Mbang Nkongo, Didier Dibong Siegfried et Marlene Ngansop

\section{Introduction}

1 La biodiversité agricole également connue sous le nom d'agrobiodiversité (AGBD) est définie comme

«la variété et la variabilité des animaux, des plantes et des micro-organismes qui sont utilisés directement ou indirectement pour la nourriture et l'agriculture, y compris les cultures, les animaux d'élevage, la forêt (aliments sauvages) et la pêche » (FAO 1999).

Elle comprend la diversité des ressources génétiques (variétés, races animales) et des espèces utilisées pour la nourriture, le fourrage, les fibres, le combustible et les médicaments. Elle inclut également la diversité des espèces non récoltées qui aident à la production (les microorganismes du sol, les prédateurs et les pollinisateurs) et celles que l'on trouve dans un environnement plus vaste qui soutiennent les écosystèmes (agricoles, pastoraux, forestiers et aquatiques) et participent à leur diversité (Torres 2012). L'AGBD est de plus en plus reconnue comme faisant partie de la stratégie plus large de diversification des moyens d'existence des petits agriculteurs (Zwart et al. 2014). Une 
abondante littérature scientifique accumulée sur l'AGBD au cours des dernières décennies témoigne de la place primordiale qu'occupe ce secteur dans la contribution à la sécurité alimentaire (SA), la réduction de la pauvreté et le développement socio-économique en Afrique et au Cameroun (Anonyme 2000, Tansey 2002, Baco et al. 2007, I.E.D. 2014). La préservation de l'AGBD est donc essentielle pour la production durable de nourriture et d'autres produits agricoles ainsi que d'autres avantages qu'elle procure à l'humanité, notamment la sécurité alimentaire, la nutrition, les moyens de subsistance, le fonctionnement des agroécosystèmes (Jackson et al. 2005). Par conséquent, son intérêt, ces dernières décennies, s'inscrit dans un cadre de conservation car il a été constaté qu'au fil des années, l'AGBD s'effrite à un rythme alarmant au profit de la monoculture qui, elle, dégrade les écosystèmes qui ont jadis servi de cadre d'épanouissement aux variétés locales (Cardoso et al. 2010, Anonyme 2014). Depuis les années 70, de nombreux travaux de recherche ont exprimé une inquiétude sur l'érosion de la diversité génétique des cultures à travers le remplacement à grande échelle des variétés traditionnelles par des variétés améliorées (Frankel 1970, Harlan 1973, Hawkes 1983, Brush 1995, Bellon 1996).

3 En Afrique sub-saharienne, près de 200 millions de personnes, soit le tiers de la population totale, souffrent de la faim, la malnutrition touche 31 millions d'enfants de moins de cinq ans (Dembélé 2010). Au Cameroun, malgré la quantité et la variété des denrées alimentaires produites, la population souffre encore de problèmes nutritionnels qui ne sont pas seulement liés au manque de nourriture mais à l'utilisation inappropriée des ressources alimentaires disponibles (Anonyme 2007, Bruinsma 2009, Nkengfack et al. 2011, Kamda et al. 2016). Dans la zone forestière humide du pays, de nombreux travaux de recherche sur l'AGBD ont montré le rôle des agroforêts à cacaoyers comme des réservoirs de biodiversité utiles pour l'alimentation, la santé, pour générer les revenus des producteurs et qui fournissent de nombreux services écosystémiques (Sonwa et al. 2007, Eyoho-Ewane 2012, Penanjo et al. 2014, Ngagoum 2015). En dehors de ces agroforêts à cacaoyers, l'AGBD dans les autres formes d'agro-écosystèmes n'a jusqu'ici été que très peu explorée. Dans la localité de Gribé, plusieurs types d'affectations de terres se juxtaposent parmi lesquels une aire protégée (parc national de Boumba Beck), des concessions forestières, une forêt communautaire, une Zone d'Intérêt Cynégétique à Gestion Communautaire et un espace agroforestier dédié aux activités agricoles et de subsistance des populations. Cette agriculture traditionnelle qui est de type itinérante sur brûlis entraine le défrichement annuel de 47 ha de forêts (Hirai 2014). Dans cette dynamique, il est intéressant de comprendre comment la diversité au sein de ces agrosystèmes varie et contribue à la sécurité alimentaire des ménages, et de manière large à la résilience de l'écosystème agricole local. En d'autres termes, les savoirs paysans traditionnels dans cette localité conservent-ils encore une agrobiodiversité adéquate pour assurer la sécurité alimentaire des ménages ? C'est pour répondre à cette question que le présent travail se propose de faire une analyse comparative de l'AGBD et des systèmes locaux de gestion au sein des petites exploitations des communautés Konabembé et Baka dans la Région de l'Est Cameroun, et les implications pour la sécurité alimentaire au niveau local. De manière spécifique, il s'est agi d'évaluer la perception locale de l'état de sécurité alimentaire des ménages, d'inventorier l'agrobiodiversité dans les différents systèmes de production des deux groupes ethniques, et analyser les implications sur la sécurité alimentaire des ménages. 


\section{Matériel et méthodes}

\section{Milieu d'étude}

4 L'étude a été réalisée à Gribé, village de l'arrondissement de Yokadouma dans le département de la Boumba et Ngoko (région de l'Est-Cameroun). Ce village est localisé par les points de coordonnées $03^{\circ} 00^{\prime} 10^{\prime \prime}$ de latitude Nord et $14^{\circ} 49^{\prime} 25^{\prime \prime}$ de longitude Est (Figure 1). Gribé fait partie du domaine de la forêt dense humide semi-caducifoliée, secteur forestier semi-caducifolié sensus stricto (Letouzey 1985). Il est arrosé par un réseau important de cours d'eaux dont les principaux sont: Adanwasa, Duosé, Mbémbé, Tilaka; ceux-ci se jettent dans la rivière Kwopkwop, affluent du fleuve Boumba, lui-même affluent de la Ngoko qui est par ailleurs affluent du grand fleuve Congo. Les sols rencontrés dans la région de Gribé sont de type ferralitique. Ce sont des sols argileux caractérisés par de fortes teneurs en oxy-hydroxydes de fer et d'alumine (Kamgang et al. 2001). Gribé subit, dans son ensemble, l'influence d'un climat équatorial de type guinéen, avec une alternance de quatre saisons annuelles bien déterminées. On y distingue : une petite saison des pluies qui va de mi-mars à fin juin; une petite saison sèche qui va de fin juin à mi-août ; une grande saison des pluies qui s'étale de mi-août jusqu'à mi-novembre et une grande saison sèche qui s'étale entre la mi-novembre et la mi-mars. La température moyenne annuelle est de $24^{\circ} \mathrm{C}$. Les précipitations moyennes annuelles sont de $145,75 \mathrm{~mm}$. Les principales espèces fauniques chassées dans les environs du village Gribé sont celles de taille moyenne telles que: les céphalophes roux bleu (Cephalophus callipygus, $C$. dorsalis, $C$.leucogaster et $C$. nigrifrons), les céphalophes à dos jaune (C. silvicultor ), et le sanglier (Potamochoerus porcus). Les plus petits animaux, tels que le céphalophe bleu (C. monticola) et les porcs-épics (Atherurus africanus), sont aussi chassés (Yasuoka 2006). Deux groupes ethniques cohabitent dans le village Gribé (les Baka et les Konabambé) et vivent, pour la plupart d'agriculture, de chasse, de cueillette, du petit commerce, du petit élevage et quelquefois de la pêche. Les populations Bantous Konabembé sont traditionnellement agricultrices tandis que les Baka, traditionnellement chasseurs-cueilleurs, adoptent et pratiquent l'agriculture depuis les années 1960 (Leclerc 2012, Oishi 2012). Le village compte 94 ménages Baka (environ 400 personnes) et 74 ménages Konabembé (environ 300 personnes) d'après le recensement effectué en 2014 (Toda 2014). 
Figure 1 : Carte de localisation du village Gribé

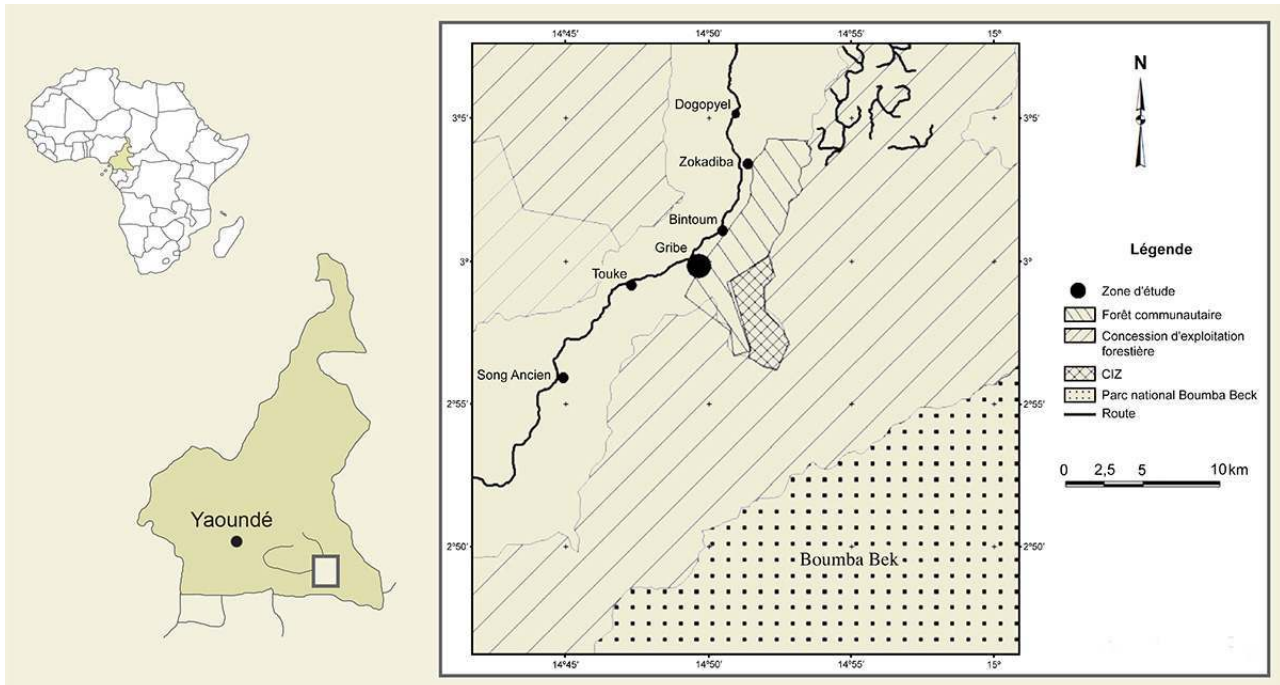

\section{Méthodologie de collecte des données}

\section{Population cible}

La présente étude a été menée auprès de 42 ménages dont 17 Baka et 25 Konabambé (Tableau 1). Les répondants constituant l'échantillon sont ceux ayant accepté volontairement de prendre part à l'étude. Leur sélection était basée sur un certain nombre de critères: ils devaient être des agriculteurs à temps plein, vivant en permanence dans la communauté, pratiquer l'agriculture vivrière (et non seulement des cultures de rente), être disposés à autoriser des visites dans leurs exploitations et partager des informations sur leurs pratiques agricoles, les problèmes et d'autres informations relatives à la sécurité alimentaire. 
Tableau 1 : Caractéristiques socio-démographiques des ménages interrogés

\begin{tabular}{|c|c|c|}
\hline Caractéristiques & Fréquence $\mathrm{N}=42$ & Pourcentage (\%) \\
\hline \multicolumn{3}{|l|}{ Groupes ethniques } \\
\hline Baka & 17 & 40 \\
\hline Konabembé & 25 & 60 \\
\hline \multicolumn{3}{|l|}{ Genre } \\
\hline hommes & 23 & 55 \\
\hline femmes & 19 & 45 \\
\hline \multicolumn{3}{|l|}{ Taille du ménage } \\
\hline$>8$ personnes & 09 & 21 \\
\hline Entre $5-8$ personnes & 17 & 40 \\
\hline$<4$ personnes & 16 & 39 \\
\hline \multicolumn{3}{|l|}{ Statut matrimonial } \\
\hline Marié & 35 & 83 \\
\hline Célibataire & 01 & 2,5 \\
\hline Veuf (ve) & 05 & 12 \\
\hline Divorcé(e) & 01 & 2,5 \\
\hline \multicolumn{3}{|l|}{ Âge } \\
\hline ] 20-35 ans ] & 24 & 59 \\
\hline ] $35-50$ ans ] & 12 & 29 \\
\hline ] 50-65 ans ] & 4 & 10 \\
\hline$>65$ ans & 1 & 2 \\
\hline \multicolumn{3}{|l|}{ Niveau d'étude } \\
\hline aucun & 18 & 43 \\
\hline primaire & 16 & 38 \\
\hline secondaire & 08 & 19 \\
\hline
\end{tabular}

Collecte des données sur la sécurité alimentaire des ménages

Des enquêtes ont été réalisées auprès des ménages cibles. L'approche pour mesurer la sécurité alimentaire est celle du guide de l'USDA (2000) basée sur des informations obtenues sur les conditions spécifiques du ménage (variation du niveau de revenus du foyer et degré d'affectation du foyer par la faim: parents uniquement, enfants uniquement, parents et enfants), d'expériences et de comportements servant d'indicateurs du degré de gravité et de sévérité des situations. Sur la base d'un questionnaire, ces enquêtes ont permis de répondre aux questions suivantes :

- les ménages disposent-ils de suffisamment de nourriture ou d'argent pour subvenir à leurs besoins essentiels de nourriture?

- si non, comment réagissent-ils face à leurs conditions?

L'USDA propose un questionnaire de mesure de la situation de sécurité alimentaire des ménages basé sur l'analyse des conditions et des réactions des ménages notamment:

- l'anxiété que le budget alimentaire des ménages ou de l'approvisionnement alimentaire puissent être insuffisants pour répondre aux besoins de base ;

- l'expérience de manquer de nourriture, sans argent pour obtenir plus;

- les perceptions par le ménage que les aliments consommés par les membres du ménage étaient de qualité ou de quantité insuffisante;

- les ajustements à l'utilisation de la nourriture normale, en remplaçant de moins en moins les aliments chers par les moins chers;

- les cas de consommation alimentaire réduite par des adultes et des enfants dans le ménage, ou les conséquences de la réduction;

- la sensation physique de la faim ou de la perte de poids;

- etc. 


\section{Collecte de données de biodiversité végétale}

8 Des relevés floristiques ont été faits dans les exploitations agricoles des répondants. Au total, 161 exploitations appartenant aux 42 ménages interrogés ont été visitées. Lors des visites dans ces exploitations, toutes les cultures présentes en champs ainsi que les arbres associés ont été identifiés et leurs utilisations renseignées. La détermination des espèces s'est faite sur le terrain avec l'aide de botanistes et des flores du Cameroun et d'Afrique centrale (Vivien \& Faure 1985, Thirakul 1983, Letouzey A. 1982, Letouzey R. 1985, Wilks \& Issembé 2000).

\section{Traitement et analyse des données}

\section{Analyse de la sécurité alimentaire des ménages}

9 L'ensemble des informations extraites du questionnaire sur la sécurité alimentaire ont été exprimées à travers une mesure appelée échelle de sécurité alimentaire. Il s'agit d'une échelle linéaire allant de 0 à 10 , telle que proposée par USDA (2000), employée afin d'apporter une estimation chiffrée du degré de sévérité de la faim subie par un ménage. Ces indicateurs correspondent aux réponses des ménages jugés dans une condition sévère/grave lors de l'enquête :

- score inférieur à 2: ménage en sécurité alimentaire présentant moins ou aucun indicateur d'insécurité alimentaire ;

- score compris entre 2 et 4 : ménage présentant une insécurité alimentaire sans famine : ici, aucune baisse de nourriture n'est enregistrée, toutefois, l'on note des variations notamment qualitatives dans les habitudes alimentaires ;

- score entre 4 et 6 : ménage présentant une insécurité alimentaire associée à une famine modérée : la baisse de ressources alimentaires est déjà manifeste chez les adultes mais ne se fait pas encore ressentir chez les enfants ;

- score supérieur à 6 : ménage présentant une insécurité alimentaire associée à une famine sévère : l'apport en nourriture affectant également les parents est si faible que les sévices de la faim sont ressentis au niveau des enfants.

10 L'interprétation de l'échelle des valeurs ainsi obtenue se fait sur la base de l'expérience directement vécue par les foyers en termes de suffisance de nourriture, et non de régime alimentaire comme le ferait un nutritionniste. Cette échelle décrit la condition des ménages pris comme entités collectives et non nécessairement la condition des membres pris individuellement.

11 Le logiciel XLSTAT a été utilisé pour les analyses multivariées (ACP et AFC) pour obtenir une répartition spatiale des nourritures consommées dans les différents ménages. Dans cette analyse, les variables sont les types d'aliments consommés dans le ménage (plantain, tubercules de manioc, macabo, pâtes alimentaires, riz, igname, viande de brousse, kohkoh, zoom, feuilles de manioc, feuilles de macabo, maïs, arachide, gombo, tomate, poisson fumé, miel, etc.).

\section{Analyse des données floristiques}

12 L'analyse des données floristiques a porté essentiellement sur la détermination des paramètres de diversité et des paramètres suivants :

- la richesse spécifique de chaque exploitation, évaluée comme étant le nombre total 
d'espèces recensées ;

- le calcul des valeurs de fréquence d'occurrence des taxons.

\section{Résultats}

\section{État de sécurité alimentaire des ménages}

La détermination des scores de sécurité alimentaire (S) des ménages selon la grille de l'USDA montre que plus de la moitié des ménages interrogés (54\%) vivent dans une situation d'insécurité alimentaire associée à une famine modérée; $29 \%$ des ménages présentent une insécurité alimentaire associée à une famine sévère; $14 \%$ des ménages présentent une insécurité alimentaire sans famine et $3 \%$ des ménages vivent en situation de sécurité alimentaire présentant moins ou aucun indicateur d'insécurité alimentaire (Figure 2).

Figure 2 : Spectre des scores de la sécurité alimentaire des ménages de Gribé

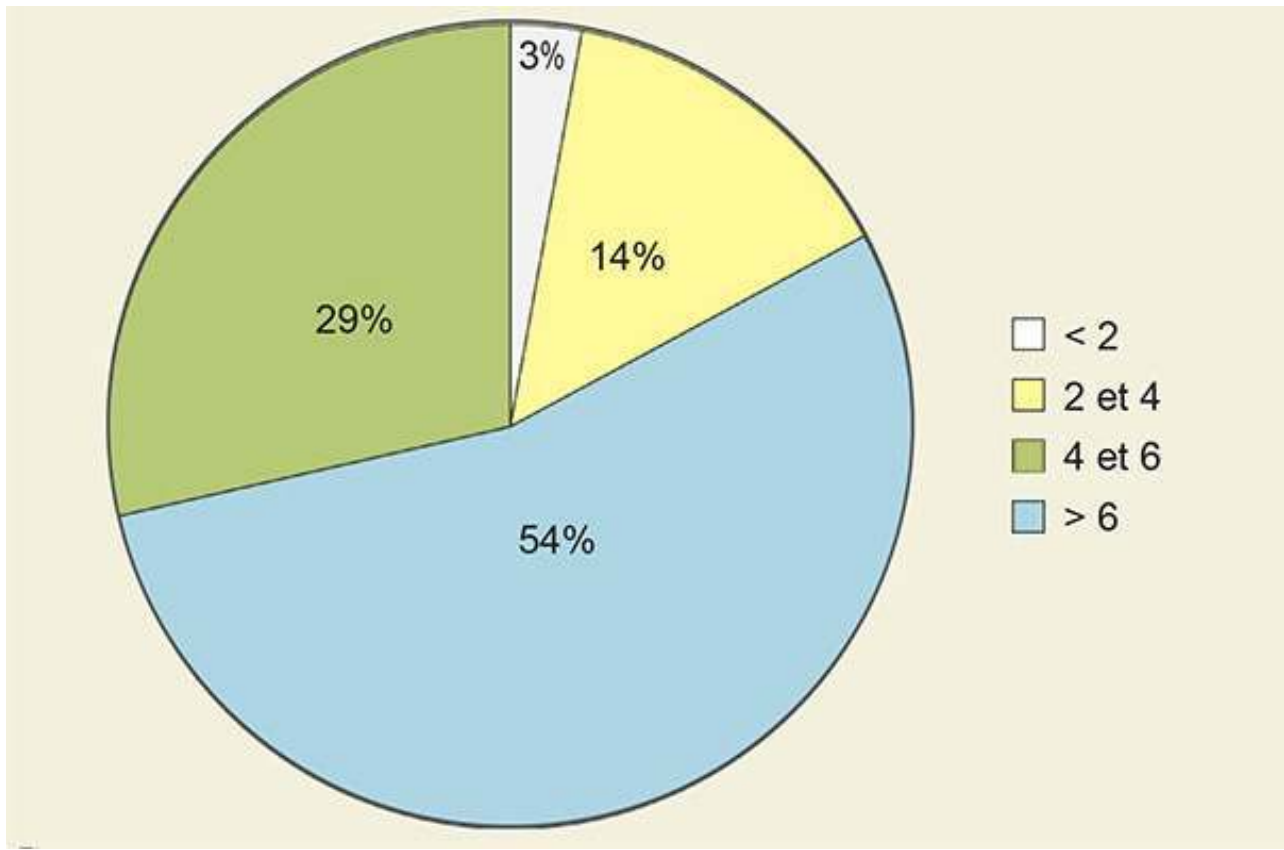

$S<2$ : ménage en sécurité alimentaire présentant moins ou aucun indicateur d'insécurité alimentaire ; $2<S<4$ : ménage présentant une insécurité alimentaire sans famine : ici, aucune baisse de nourriture n'est enregistrée, toutefois, l'on note des variations notamment qualitatives dans les habitudes alimentaires ; $4<S<6$ : ménage présentant une insécurité alimentaire associée à une famine modérée : la baisse de ressources alimentaires est déjà manifeste chez les adultes mais ne fait pas encore ressentir chez les enfants ; S> 6 : ménage présentant une insécurité alimentaire associée à une famine sévère : l'apport en nourriture affectant également les parents est si faible que les sévices de la faim sont ressentis au niveau des enfants.

Quelques disparités existent entre les groupes ethniques quant à la perception de la sécurité alimentaire. Ainsi, $4 \%$ de ménages Konabembé vivent dans une situation de sécurité alimentaire avec moins ou aucun indicateur d'insécurité alimentaire (Figure 3). Pour les Baka $20 \%$ contre $28 \%$ de Konabembé vivent en situation d'insécurité alimentaire sans famine. Quant aux ménages présentant une insécurité alimentaire associée à une famine modérée, les Baka et Konabembé partagent la même proportion soit $40 \%$ pour 
chaque groupe ethnique. $28 \%$ de ménages Konabembé vivent en situation d'insécurité alimentaire associée à une famine sévère contre $40 \%$ de ménages Baka.

Figure 3 : État de perception de la sécurité alimentaire des ménages par groupe ethnique

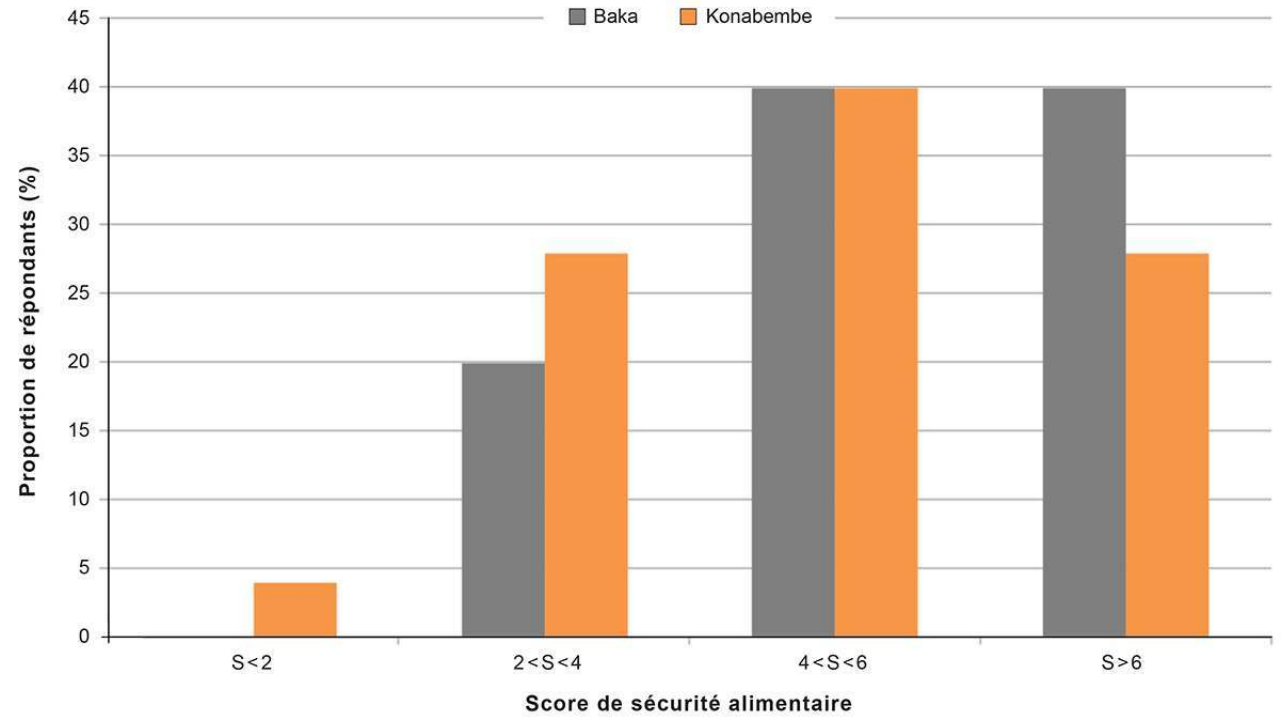

$\mathrm{AMC}=$ achat aux autres membres de la communauté $; \mathrm{AM}=$ achat sur le marché

La nourriture consommée dans les ménages a pour principale provenance le champ (48\% ) et minoritairement d'autres sources notamment la forêt $(8 \%)$. Celle consommée et provenant de l'achat aux autres membres de la communauté, l'achat sur le marché mais aussi celle provenant de dons comptent respectivement pour $14 \%, 17 \%$ et $13 \%$ (Figure 4).

Figure 4 : Provenance de la nourriture consommée dans les ménages

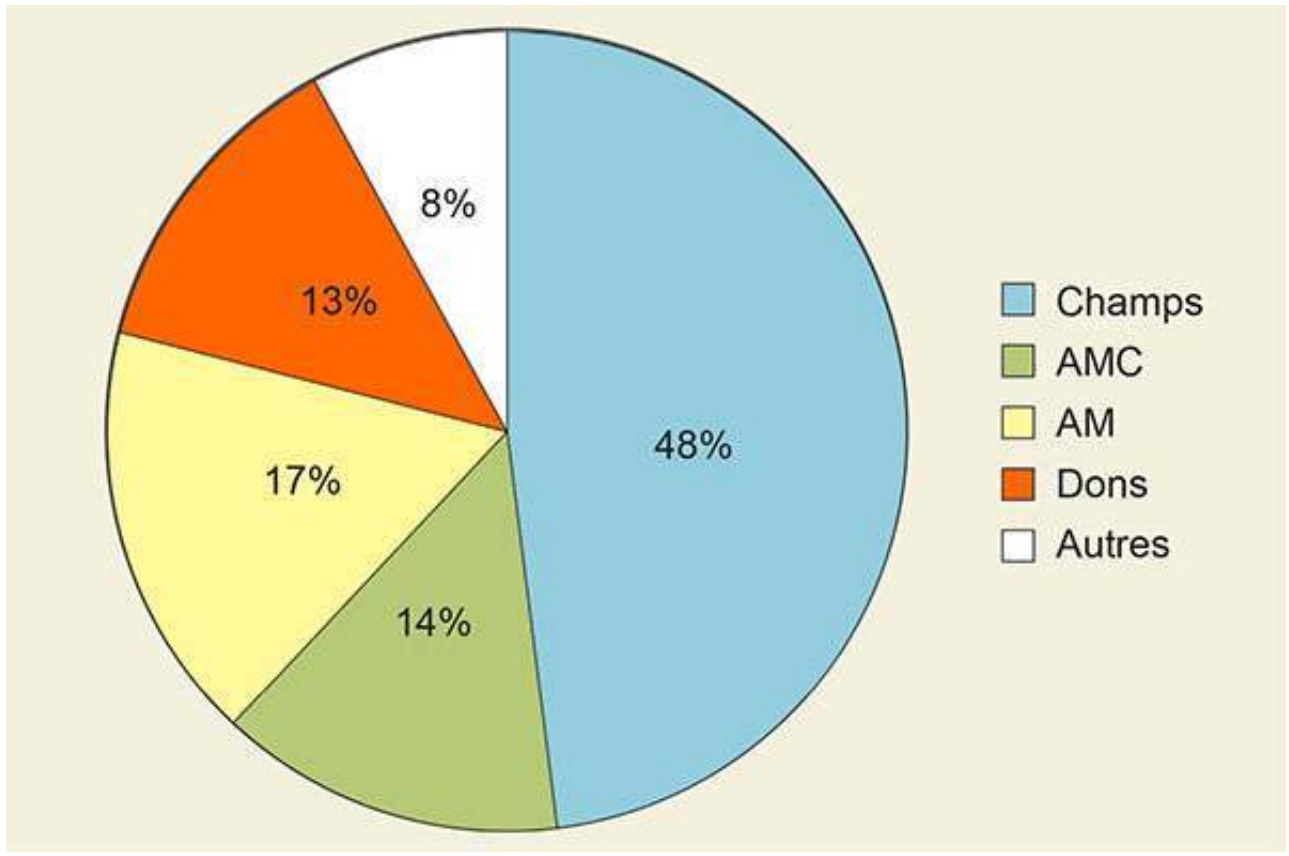


L'analyse en composante principal (ACP) ci-dessous présente l'ordre de fréquence de consommation des aliments dans les ménages (Figure 5), fréquence liée à la préférence alimentaire. Selon le cercle de corrélation, les axes factoriels F1 et F2 représentent $51,60 \%$ d'inertie totale. Ces axes factoriels F1 et F2 permettent de différencier quatre groupes. Le groupe I constitué uniquement du plantain (Musa sapientum). Le groupe II rapprochant les fréquences 2 à 6 , est constitué des tubercules et feuilles de manioc ( Manihot esculenta); macabo (Xanthosoma sagittifolium); kohkoh (Gnetum africanum) et viande de brousse. Le groupe III est constitué de péké (Irvingia gabonensis) ; zoom (Solanum nigrum) ; gombo (Abelmoschus esculentus); pâtes alimentaires, feuilles de macabo, maïs (Zea mays); arachide (Arachis hypogaea) et riz. Le groupe IV constitué des aliments tels l'igname (Dioscorea rotundata), l'igname sauvage localement appelé bâ en langue Baka (Dioscorea mangenotiana), le miel, le poisson fumé, la sardine, le concombre (Citrullus sp.), sapa, chenille, ndolè (Vernonia amygdalina), mbi, dandou, patate douce (Ipomea batatas), tomate (Solanum lycopersicum), souma, champignon (Termitomyces sp.), escargot et banane douce ( Musa indica) consommés chaque fois que l'occasion se présente.

Figure 5 : Variation des aliments consommés par ordre de leur préférence dans les ménages

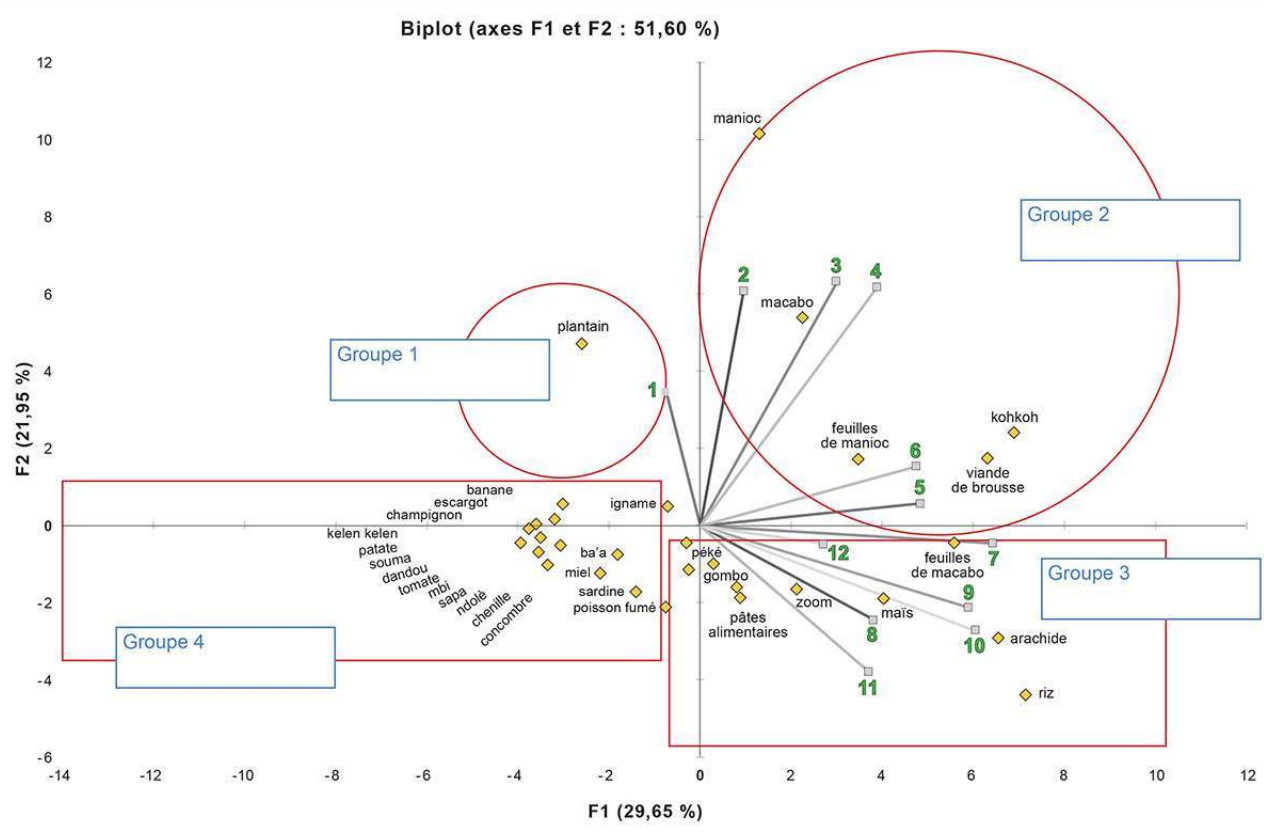

\section{Différents systèmes d'utilisation des sols}

17 La figure 6 donne un aperçu des différents types d'utilisation des sols dont dispose chaque ménage. Cinquante-sept (57\%) sont des associations de champs vivriers et d'agroforêt cacaoyère, 31 \% pour les associations champs vivriers et jardin de case. Les champs vivriers seuls ou les associations des trois précédents types représentent chacun $6 \%$ (Figure 6). 
Figure 6 : Proportion des différents types d'exploitations agricoles des répondants

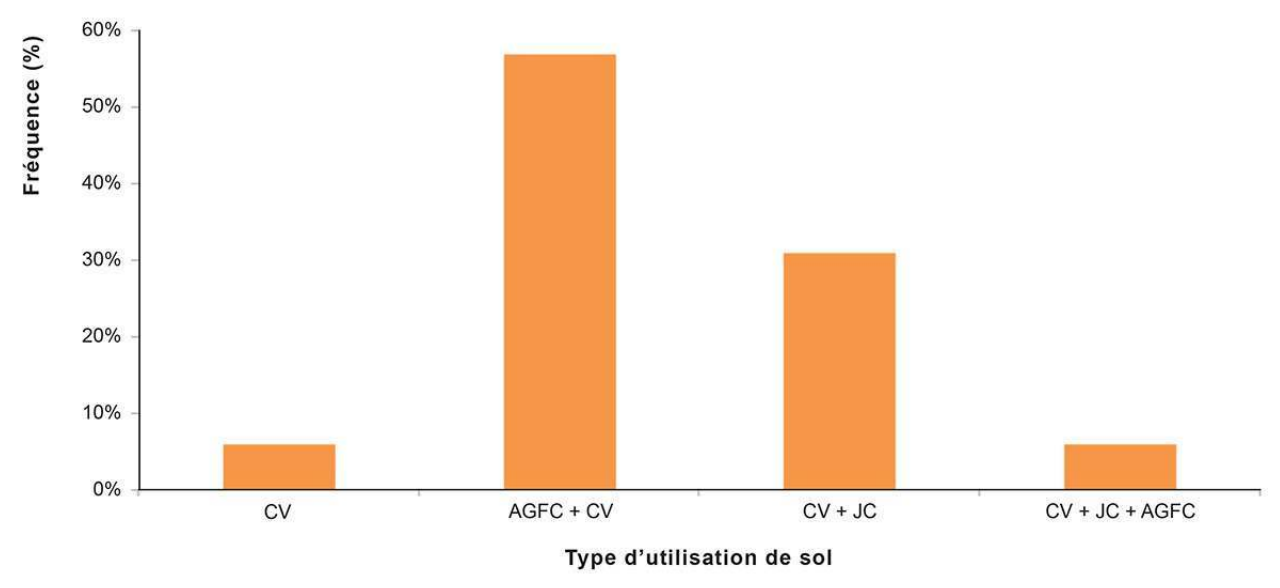

$\mathrm{CV}=$ champs vivriers, $\mathrm{AGFC}=$ agroforêt à base de cacaoyers, $\mathrm{JC}=$ jardin de case

Les pratiques culturales sont presque les mêmes dans les ménages enquêtés. Toutefois, deux variantes ont été identifiées. Dans la première, les planteurs après préparation du champ sèment au même moment le cacao, le plantain et les autres vivriers. Les rejets de plantain serviront de support et d'ombrage pour les futurs plants de cacaoyer après la récolte des cultures vivrières saisonnières. Pour la deuxième variante, après avoir défriché la forêt, les agriculteurs plantent les rejetons de bananier plantain avant de passer au brûlis. Les personnes effectuant cette pratique ont confié qu'elles le font pour rattraper le temps lorsque le champ n'a pas été brûlé à temps. Ainsi, après passage du feu, les bananiers germent en premier, ce qui permet de planter immédiatement le cacao à côté. D’après eux, le rendement de cette pratique est bien meilleur que le précédent.

\section{Composition et diversité floristiques des agrosystèmes}

19 Les relevés floristiques dans les exploitations agricoles ont permis de recenser un total de 170 espèces regroupées en 61 familles ; 159 ont été déterminées au niveau spécifique et 11 au niveau du genre.

20 Les 10 familles les plus riches en espèces sont les Sterculiaceae (11 espèces), les Euphorbiaceae (10 espèces), les Solanaceae ( 9 espèces), les Annonaceae et les Mimosaceae (8 espèces), les Caesalpiniaceae, les Olacaceae et les Ulmaceae ( 7 espèces), les Anacardiaceae et les Rubiaceae ( 6 espèces).

21 Parmi les 170 espèces, 34 sont des plantes cultivées appartenant à 20 familles. L'analyse de cette biodiversité cultivée montre que l'espèce la plus fréquente est Manihot esculenta (12,9\% d'occurrence), suivie de Musa sapientum (12\%), Xanthosoma sagittifolium (9,5\%), Theobroma cacao (8,1\%), Carica papaya (7,2 \%), Capsicum frutescens (6,9\%), Zea mays (6,9\%), Arachis hypogaea (6,1\%), Musa paradisiaca (4,9\%) et Nicotina tabacum (4\%). Le famille des Solanaceae est représentée par neuf espèces et est celle renfermant le plus grand nombre d'espèces cultivées. Il s'agit de : Capsicum frutescens, Nicotina tabacum, Solanum lycopercium, Solanum macrocarpon, Solanum melogena, Solanum nigrum et Solanum tuberosum (Figure 7). 
Figure 7 : Histogramme des fréquences des espèces cultivées

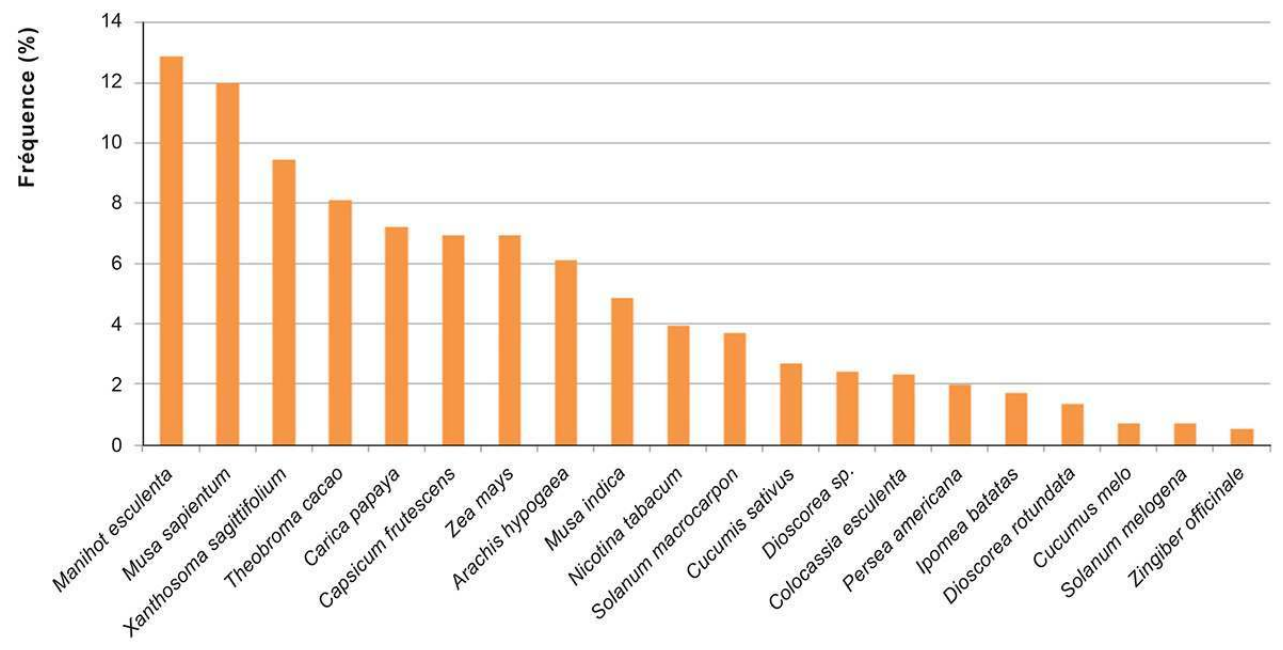

Les espèces forestières sauvages accompagnant les cultures en champ sont au nombre de 136 et réparties dans 51 familles botaniques. Les plus riches en espèces sont les Sterculiaceae avec 10 espèces (Figure 8), les Euphorbiaceae et les Annonaceae (8 espèces), les Ulmaceae, les Olacaceae, les Mimosaceae et les Caesalpiniaceae ( 7 espèces). Les espèces les plus fréquentes sont: Musanga cecropioides (7,5\%), Gnetum sp.(6,1\%), Trema orientalis $(5,3 \%)$, Ricinodendron heudelotii (4 \%), Albizia glaberrina (3,4 \%), Pycnanthus angolensis $(3,3 \%$ ), Terminalia superba (3,3\%) et Pentaclethra macrophylla (3,2 \%).

Figure 8 : Histogramme de fréquence des espèces accompagnant les cultures en champ

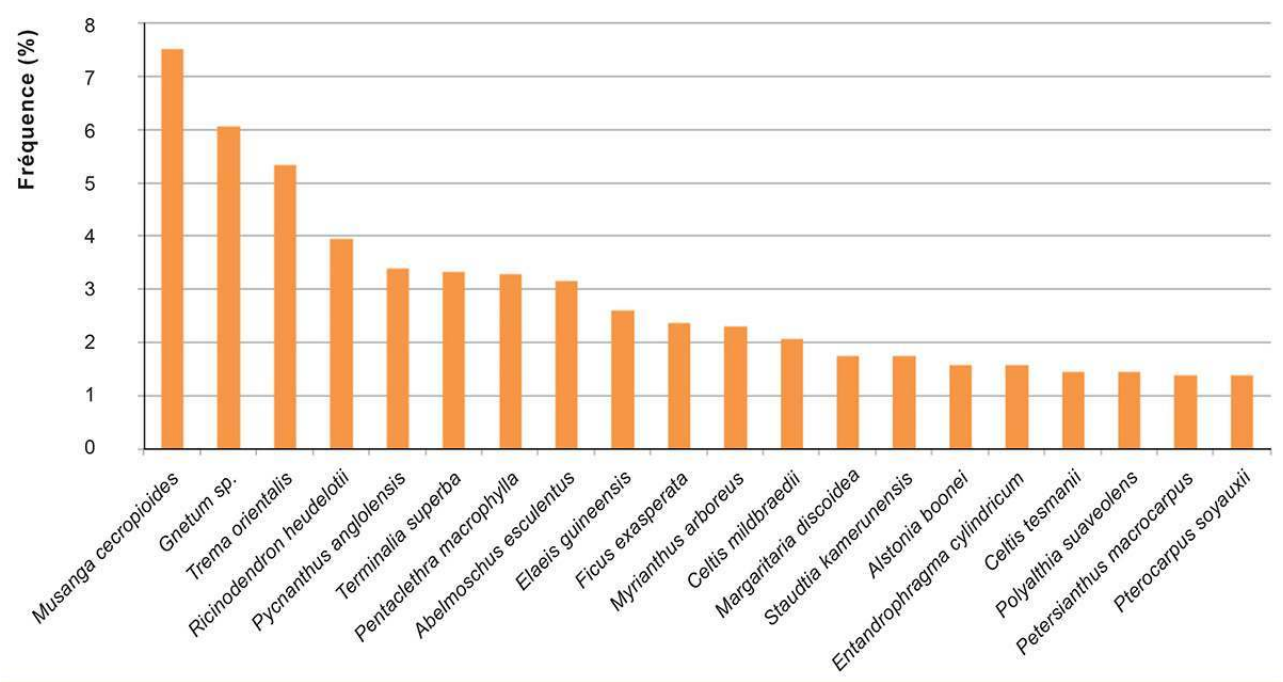

\section{Comparaison de la composition floristique des exploitations des deux groupes ethniques}

Parmi les 170 espèces recensées, 112 dont 21 cultivées forment le fond floristique commun aux exploitations Baka et Konabembé. Cette communauté botanique masque tout de même quelques fines différences. Dix espèces sont présentes uniquement dans les exploitations Konabembé. Il s'agit de: Allium cepa, Cucumus melo, Cymbopogon citratus, 
Dioscorea rotundata, Phasoelus vulgaris, Solanum lycopercium, Solanum torvum, Solanum tuberosum, Vernonia amygdalina et Zingiber officinale (Tableau 2).

Tableau 2 : Fréquence des espèces cultivées selon le groupe ethique

\begin{tabular}{|c|c|c|c|}
\hline \multirow{2}{*}{$\mathbf{N}^{\circ}$} & \multirow{2}{*}{ Especes } & \multicolumn{2}{|c|}{ Fréquence des espèces selon le groupe ethique } \\
\hline & & B & $\mathrm{k}$ \\
\hline 1 & Musa sapientum & 17,32 & 9,47 \\
\hline 2 & Manihot esculenta & 16,76 & 11,07 \\
\hline 3 & Xanthosoma sagittifolium & 11,73 & 8,40 \\
\hline 4 & Theobroma cacao & 8,10 & 8,13 \\
\hline 5 & Carica papaya & 8,10 & 6,80 \\
\hline 6 & Zea mays & 6,42 & 7,20 \\
\hline 7 & Capsicum frutescens & 5,87 & 7,47 \\
\hline 8 & Arachis hypogaea & 3,63 & 7,33 \\
\hline 9 & Musa indica & 3,63 & 5,47 \\
\hline 10 & Nicotina tabacum & 3,63 & 4,13 \\
\hline 11 & Dioscorea sp & 3,07 & 2,13 \\
\hline 12 & Persea americana & 3,07 & 1,47 \\
\hline 13 & Ipomea batatas & 1,96 & 1,60 \\
\hline 14 & Solanum macrocarpon & 1,12 & 4,93 \\
\hline 15 & Colocassia esculenta & 1,12 & 2,93 \\
\hline 16 & Dioscorea rotundata & 1,12 & 1,47 \\
\hline 17 & Cucumis sativus & 0,84 & 3,60 \\
\hline 18 & Solanum melogena & 0,56 & 0,80 \\
\hline 19 & Saccharum afficinarum & 0,56 & 0,40 \\
\hline 20 & Ananas comosus & 0,28 & 0,40 \\
\hline 21 & Cocos nucifera & 0,28 & 0,13 \\
\hline 22 & Dacryodes edulis & 0,28 & 0,13 \\
\hline 23 & Manguifera indica & 0,28 & 0,00 \\
\hline 24 & Musa sapientum & 0,28 & 0,00 \\
\hline 25 & Cucumus melo & 0 & 1,07 \\
\hline 26 & Zingiber officinale & 0 & 0,80 \\
\hline 27 & Phaseolus vulgaris & 0 & 0,67 \\
\hline 28 & Solanum torvum & 0 & 0,67 \\
\hline 29 & Solanum Iycopercium & 0 & 0,53 \\
\hline 30 & Allium cepa & 0 & 0,13 \\
\hline 31 & Cymbopogon citratus & 0 & 0,13 \\
\hline 32 & Discorea rotundata & 0 & 0,13 \\
\hline 33 & Solanum tuberosum & 0 & 0,13 \\
\hline 34 & Vernonia amygdalina & 0 & 0,13 \\
\hline
\end{tabular}

Sur les 136 espèces forestières accompagnant les cultures, 91 sont communes aux exploitations des deux groupes ethniques. Dix-neuf sont présentes uniquement dans les exploitations Konabembé. Il s'agit de: Homalium letestui, Trichoscypha acuminata, Lasiodiscus mannii, Angylocalyx pynaertii, Desplatsia dewevrei, Anopyxis klaineana, Bridelia grandis, Chytranthus atroviolaceus, Dicranolepis disticha, Funtimia elastic, Magaritaria discoidea, Maranthes labra, Mildbraediodendron excelsum, Neosloetiopsis kamerunensis, Ochtocasmus africanus, Ophiobotrys zenkeri, Rinorea lepidobotrys, Stombosia pustulata et Xylopia phloidora. Vingt-quatre espèces sont présentes uniquement dans les exploitations Baka. Il s'agit de : Milicia excelsa, Sterculia tragacantha, Picralima nitida, Triplochiton scleroxylon, Lannea welwitschii, Pseudospondis microcarpa, Psychotria peduncularis, Brenania brieyi, Bridelia grandis , Cola sp., Copaifera mildbraedii, Cucumus melo, Dialium dinklagei, Drypetes sp., Enantia chlorantha, Eremomastax speciosa, Gambeya boukokoensis, Hallea stipulosa, Rinorea sp., Spataudtia companulata, Sterculia tragancantha, Stombosiopsis sp., Strombosia pustulata et Strombosiopsis sp.

\section{Discussion}

\section{Profil de sécurité alimentaire des ménages}

Les résultats montrent que les ménages dans la zone connaissent une insécurité alimentaire associée à des degrés variables de famine. Au Cameroun en général et dans les zones rurales en particulier, l'insécurité alimentaire est de plus en plus une réalité. 
Malgré la quantité et la variété des denrées alimentaires produites, la population souffre encore de problèmes nutritionnels qui ne sont pas seulement liés au manque de nourriture mais également à l'utilisation inappropriée des ressources alimentaires disponibles (Anonyme 2007, Bruinsma 2009, Nkengfack et al. 2011, Kamda et al. 2016). Le Programme Alimentaire Mondiale et la FAO, dans une étude sur l'analyse de la vulnérabilité et de la sécurité alimentaire au Cameroun en 2011, montrent qu'en tout temps, au moins $30 \%$ de ménages camerounais en zones urbaine et rurale demeurent vulnérables à l'insécurité alimentaire (WFP et FAO 2011). Dans la présente étude, les populations Baka sont les plus affectées. Ce groupe ethnique, traditionnellement des chasseurs-cueilleurs, connaît depuis quelques décennies, des transitions dans ses modes d'alimentation. Plusieurs études anthropologiques montrent, en effet, qu'au contact de leurs voisins Bantous, leurs activités de subsistance ont évolué considérablement du statut de chasseur-cueilleur à celui d'agriculteur (Bahuchet 1985, Ichikawa 1991, Kitanishi 1995). Dans ces circonstances, les Bakas adoptent progressivement l'agriculture et la quasi-totalité d'entre eux ont maintenant leurs propres champs. Ces résultats montrent ainsi qu'en dépit de cette relative adoption des pratiques culturales, les peuples Bakas sont encore loin d'assurer leur propre sécurité alimentaire. Kitanishi (2003) montrait, à cet effet, que les Baka, bien qu'ayant adopté l'agriculture dans les années 1950, celle-ci reste non planifiée et aléatoire, en raison du temps considérable qui reste alloué à la chasse et à la cueillette, ce qui les rend encore très dépendants des vivres obtenus auprès des agriculteurs bantous voisins, en échange de produits forestiers ou de travaux agricoles. Au contact des Konabembé, ils apprennent à cultiver les produits les plus courants dans la région, notamment le plantain, le manioc, le maïs, les arachides. Leur alimentation est faible voir pauvre en légumes. Cette alimentation pauvre en légumes a un impact fort considérable sur la sécurité alimentaire de ce peuple. Sur les légumes rencontrés dans le village Gribé, les Baka ne consomment que du kohkoh (Gnetum spp.). Les feuilles de manioc (Manihot esculenta), de macabo (Xanthosoma sagittifolium) et le zoom (Solanum nigrum) ne font pas partie de leurs habitudes alimentaires. Or, ces trois légumes possèdent chacun un important potentiel en termes de nutriments et sont parmi les légumes-feuilles tropicaux les plus consommés en Afrique sub-saharienne (Kahane et al. 2005, Maundu et al. 2009, PROTA 2004).

\section{Caractéristiques de la biodiversité agricole locale}

Un total de 170 espèces a été recensé dans les exploitations agricoles. La famille la plus riche est celle des Solanaceae qui regroupe par ailleurs la grande majorité des légumefeuilles tropicaux cultivée (Samuels 2015). Parmi les espèces accompagnant les cultures, l'on note une grande fréquence des Sterculiaceae, Euphorbiaceae, Annonaceae, Ulmaceae, Olacaceae, Mimosaceae et Caesalpiniaceae. Ce résultat reflète les caractéristiques floristiques de ce massif forestier telles que décrites par Nkongmeneck (1996) et Tajeukem et al. (2014). Les espèces accompagnant les cultures sont conservées en champs en raison des divers services qu'elles rendent notamment les services de prélèvement (alimentation et médicament) mais aussi ceux de régulation (ombrage).

En fonction des groupes ethniques, il apparaît très peu de différence en termes d'espèces cultivées. Toutes espèces considérées, il semble toutefois que les exploitations Baka soient plus riches en espèces que les exploitations Konabembé. Ceci traduit le fait qu'au moment du défrichement pour la création des parcelles agricoles, les Baka détruisent moins la 
biodiversité et conservent plus d'arbres dans leurs exploitations. Au-delà de l'intérêt utilitaire que présentent ces espèces ainsi préservées, plusieurs autres raisons justifient ce fait. Dans certains cas, les répondants ont expliqué cela par le manque de matériel pour l'abattage (tronçonneuse en l'occurrence) ou par le grand diamètre de l'arbre ou la dureté de son bois qui rend l'abattage à la hache pénible.

Toutefois, en zone rurale, plusieurs facteurs autres que ceux testés dans le cadre de cette étude peuvent avoir un impact sur la diversité au sein des agroécosystèmes. Dans les paysages d'Usambara à l'est de la Tanzanie, Powell et al. (2017) ont montré, en effet, que des facteurs tels que les capacités financières du ménage, la diversité des moyens d'existence des populations, la saisonnalité des cultures, la taille du ménage, le genre, influencent leur faculté à cultiver une gamme plus ou moins variée de ressources pour assurer leur sécurité alimentaire. Tesfaye (2013) démontrait aussi en Éthiopie que la taille des champs, la proximité des marchés avait un impact sur la richesse et la diversité des cultures en champs.

\section{Conclusion}

L'étude a permis de comprendre que les populations du village Gribé sont toutes agricultrices et pratiquent une agriculture polyvariétale. Cette agriculture est une solution face aux problèmes de famine et d'insécurité alimentaire. Bien qu'ayant une multiplicité d'espèces cultivées, les populations du village Gribé restent affectées par tous les niveaux d'insécurité alimentaire tels que décrits par le guide USDA. L'insécurité alimentaire dont sont victimes les Baka est le résultat de leur transition du mode de vie de chasseur-cueilleur à la pratique d'une agriculture de subsistance, d'une agriculture peu diversifiée et pauvre en nutriments de qualité mais provient aussi de leur faible niveau d'intégration au petit marché local. Pour les Konabembé, l'insécurité alimentaire est due au manque de nutriments énergétiques de qualité. Malgré la relative intégration du petit marché, les Konabembé se heurtent au problème d'accessibilité car les prix des produits aux valeurs nutritives de qualité ne sont pas à la portée de tous. L'étude réalisée à Gribé a permis de recenser 34 espèces cultivées et 136 espèces compagnes. Ces dernières sont à la base de diverses fonctions de soutien, de régulation et de prélèvement. Le groupe ethnique et l'âge sont les principaux déterminants de la variation de la biodiversité agricole dans la zone d'étude. L'étude démontre que, du point de vue quantitatif, la petite agriculture locale est satisfaisante en tant que productrice de nourriture mais du point de vue qualitatif, elle a du mal à répondre aux exigences d'une alimentation équilibrée.

Cette étude a été réalisée grâce à l'appui financier du Forest Savannah Sustainability Project (FOSAS) du programme SATREPS, un programme financé par Japan Science and Technology Organization (JST), Japan International Cooperation Agency (IICA) et le Gouvernement de la République du Cameroun, à qui nous exprimons notre profonde gratitude. Nos remerciements vont également à l'endroit des populations du village Gribé pour leur hospitalité et leur assistance. 


\section{BIBLIOGRAPHIE}

Anonyme 2000 - Gestion de l'agrobiodiversité dans les zones rurales. GTZ : 2-4.

Anonyme 2007 - Analyse globale de la sécurité alimentaire et de la vulnérabilité. République au Cameroun, PAM, Cameroun : 4-5.

Baco M.N., Biaou G., Pinton F. \& Lescure J.-P. 2007 - Les savoirs paysans traditionnels conserventils encore l'agro-biodiversité au Bénin? Biotechnol. Agron. Soc. Environ. 11 (3) : 201-210.

Bahuchet S. 1985 - Les Pygmées Aka et la Forêt Centrafricaine. Paris, SELAF, 638 p. (Ethnosciences ; 1).

Bellon M.R. 1996 - The dynamics of crop infraspecific diversity: a conceptual framework at the farmer level 1. Economic Botany 50 : 26-39.

Bruinsma J. 2009 - The resource outlook to 2050: By how much do land, water use and crop yields need to increase by 2050? Proceedings of the expert meeting on how to feed the world in 2050. Food and Agriculture Organization of the United Nations, Economic and Social Development Department. 33 p. http://www.fao.org/fileadmin/templates/esa/Global_persepctives/Presentations/ Bruinsma_pres.pdf.

Brush S.B. 1995 - In situ conservation of landraces in centres of crop diversity. Crop Science 35 : 346-354.

Cardoso T., Pereira L.E. \& Emperaire L. 2010 - Rôle des dynamiques spatio-temporelles dans la conservation de l'agrobiodiversité des systèmes agricoles amérindiens du bas Rio Negro (Amazonas, brésil). Actes du colloque Innovation and Sustainable Development in Agriculture and Food (ISDA 2010), Montpellier, France, 28/06 au 01/07/2010, 12 p.

Dembélé G. 2010 - Connaissances et pratiques des mères par rapport à la nutrition et la santé des enfants de 6 à 59 mois dans le district sanitaire de Selingue. Thèse de Docteur en Pharmacie. Faculté de Médecine, de Pharmacie et d'Odonto-Stomatologie, Université de Bamako (Mali), 113 p.

Eyoho-Ewane S.N. 2012 - Écologie et caractérisation des agroforêts à base de cacaoyer du sud-ouest Cameroun (département de la Meme). Mémoire de fin d'études de DESS en agroforesterie, Université de Yaoundé I, $67 \mathrm{p}$.

FAO 1999 - Sustaining the multiple functions of agricultural biodiversity. Background Papers, conference on the multifunctional character of agriculture and land. Maastricht, FAO Netherlands. $42 \mathrm{p}$.

Frankel J. 1970 - Managing global genetic resources: Agricultural crops issues and policies. Washington, DC., The National Academies Press, 173 p.

Harlan J.R. 1973 - Genetics resources of some major fields crops in Africa. insurvey of crop genetic resources in their centres of diversity. Rome, Italy, FAO- IBP : 45-64.

Hawkes J.R. 1983 - The diversity of crop plants. Cambridge, MA, Harvard University press, 184 p.

Hirai M. 2014 - Agricultural land use, collection and sales of non-timber forest products in the Agroforest Zone in southeastern Cameroon. African Study Monographs Supplementary Issue 49 : 167-200. 
Ichikawa M. 1991 - The impact of commoditization on the Mbuti of eastern Zaire. In : Peterson N. \& Matsuyama T. (Ed.) Cash, Commoditization and Changing Foragers. Senri Ethnological Studies 30 : 135-162.

Innovation Environnement Développement 2014 - Agrobiodiversité et sécurité alimentaire. Revue sur l'Agriculture durable à faibles apports externes 30 (1) : 4-8.

Jackson L., Kamal B., Pascual U. \& Perrings C. 2005 - L"agro-biodiversité : un nouvel agenda pour la science de la biodiversité à l'appui du développement durable des agroécosystèmes. Diversitas Report 4 : 14-16.

Kahane R., Temple L., Brat P. \& De Bon H. 2005 - Légumes feuilles des pays tropicaux : diversité, richesse économique et valeur sante dans un contexte très fragile. Colloque Angers 7-9 septembre 2005 Les légumes : un patrimoine à transmettre et à valoriser Thème III : Utilisation et perception. $9 \mathrm{p}$.

Kamda A., Achu Loh M., Kouebou C., Ponka R. \& Yadang G. 2016 - Compilation study of Central African agrobiodiversity: Case of 20 Roots, Tubers and their products in Cameroon. Poster presented at the World Congress on Roots and Tuber Crops, 18-22 january 2016, Nanning Guanxi, China.

Kamgang K.B., Ekodeck V.G.E. \& Njilah K.I. 2001 - Évolution géochimique des formations latéritiques dans l'écosystème péri forestier du sud-est Cameroun : le site de Kandara. African Journal of Science and Technology 2 (1) : 19-32.

Kitanishi K. 1995 - Seasonal changes in the subsistence activities and food intake of the Aka hunter-gatherers in northeastern Congo. African Study Monographs, 16 (2) : 73-118.

Kitanishi K. 2003 - Cultivation by the Baka hunter-gatherers in the tropical rain forest of central Africa. African Study Monographs Supplementary Issue 28 : 143-157.

Leclerc C. 2012 - Agriculture Adopted by the Baka Pygmies in Cameroon. Social Dynamic and Structural Continuity. Quae, 246 p.

Letouzey A. 1982 - Manuel de botanique forestière. Afrique tropicale. CTFT, Tomes 2A et 2B, 461 p.

Letouzey R. 1985 - Carte phytogéographique du Cameroun et notice. Toulouse, Inst. Carte. Internat. Vegetation, $240 \mathrm{p}$.

Maundu P., Achigan-Dako E.\& Morimoto Y. 2004 - Biodiversity of African Vegetables. In : Shackleton C.M., Pasquini M.W. and Drescher A.W. African Indigenous Vegetables in Urban Agriculture. London, Earthscan : 65-104.

Ngagoum V. 2015 - Évaluation de la productivité et des usages des espèces végétales dans les complexes agroforestiers à base de cacaoyers. Conference présentation, Africa, climate change and sustainable development. What is at stake at Paris and beyong, 28-30 octobre 2015, Victoria Falls, Zimbabwe, $11 \mathrm{p}$.

Nkengfack A.E., Mvot Akak C., Mbazoa D., Tu P.F. \& Lei L.D. 2011 - New coumarin glycosides from the leaves of Diospyros crossiflora (Heirn), Fitoterapia 81 : 873-877.

Nkongmeneck B.A. 1996 - The Boumba Beck \& Nki forest reserve: Botany and Ethnobotany. Rapport technique: WWF CARPO Eds., 212 p.

Oishi T. 2012 - Cash crop cultivation and interethnic relations of the Baka hunter-gatherers in southeastern Cameroon. African Study Monographs Suppl. 43 : 115-136.

Penanjo S, Fongnzossie F.E., Nkongmeneck B.A. \& Kemeuze V.A. 2014 - Species composition and abundance of non-timber forest products in different-aged cocoa agroforests in South-Eastern Cameroon. African Study Monographs 49 : 47-67. 
Powell B., Kerr R.B, Young S.L. \& Johns T. 2017 - The determinants of dietary diversity and nutrition: ethnonutrition knowledge of local people in the East Usambara Mountains, Tanzania. Journal of Ethnobiology and Ethnomedicine 13 : 23. doi: 10.1186/s13002-017-0150-2.

PROTA 2004 - Ressources végétales de l'Afrique tropicale. Volume 2 : Légumes. Grubben G.J.H. \& Denton O.A. (Ed.). Wageningen, Fondation PROTA/Backhuys Publishers/CTA, 737 p.

Samuels J. 2015 - Biodiversity of Food Species of the Solanaceae Family: A Preliminary Taxonomic Inventory of Subfamily Solanoideae. Resources $4: 277-322$.

Sonwa D., Nkongmeneck A.B., Weise S., Tchatat M., Adesina A. \& Janssens M.J. 2007 - Diversity of plants in cocoa agroforests in the humid forest zone of Southern Cameroon. Biodivers. Conserv. 16 : 2385-2400.

Tajeukem V.C, Fongnzossie E., Kemeuzeu V.A. \& Nkongmeneck B.-A. 2014 - Vegetation Structure and Species Composition at the Northern Periphery of the Boumba -Bek National Park, Southeastern Cameroon: African Study Monographs Suppl. 49 : 13-46.

Tansey G. 2002 - Commerce, propriété intellectuelle, alimentation et diversité biologique. Document de discussion. Eds Quaker Peace \& Service, $32 \mathrm{p}$.

Tesfaye A. 2013 - Determinants of crop diversity and composition in Enset-coffee agroforestry homegardens of Southern Ethiopia. Journal of Agriculture and Rural Development in the Tropics and Subtropics 114 (1) : 29-38.

Thirakul S. 1983 - Manuel de dendrologie, Cameroun. Québec, Canada, Groupe Poulin, Thériault Ltée, $640 \mathrm{p}$.

Toda M. 2014 - People and social organizations in Gribe, southeastern Cameroon. African Study Monographs Supplementary Issue 49 : 137-166.

Torres A.C. 2012 - Revue bibliographique des outils pour la mesure des relations entre agrobiodiversité et nutrition. mémoire de Master, Université Paris Sud, 73 p.

USDA 2000 - Guide to Measuring Household Food Security. Office of Analysis, Nutrition, and Evaluation Food and Nutrition Service, USDA, $82 \mathrm{p}$.

Vivien J. \& Faure J.-J. 1985 - Arbres des forêts denses d'Afrique centrale. Espèces du Cameroun. Paris, France, Ministère des Relations extérieures, Agence de coopération culturelle et technique, $565 \mathrm{p}$. Wilks C. \& Issembé M.Y. 2000 - Guide pratique d'identification des arbres de la guinée équatoriale, région continentale. Projet CUREF Bata Guinée Équatoriale 546 p.

WFP \& FAO 2011 - Cameroon: Comprehensive Food Security and Vulnerability Analysis. Yaoundé, Cameroon, WFP and FAO, $10 \mathrm{p}$.

Yasuoka H. 2006 - The sustainability of duiker (Cephalophus spp.) hunting for the baka huntergatherers in southeastern Cameroon. African Study Monographs 33 : 95-120.

Zwart G., Doornbos S. \& Douma W. 2014 - Agriculture, biodiversité et communautés : une équation plausible? Revue Agriculture durable à faibles apports externes 30 (1) : 6-7.

\section{RÉSUMÉS}

L'agrobiodiversité joue un rôle important comme source de revenus, levier pour la résilience des systèmes agricoles aux changements climatiques, réservoir de nutriments, base de divers services écosystémiques tels que la pollinisation, la fertilité des sols et la régulation des cycles 
hydriques. Malgré ce potentiel, elle connaît une érosion importante, ces dernières années. Ce travail fait une caractérisation de l'agro-biodiversité au sein des paysages forestiers utilisés par les groupes ethniques Konabembé et Baka dans le Sud-Est Cameroun, et les implications pour la sécurité alimentaire des ménages. Des enquêtes ethnobotaniques ont été réalisées auprès de 42 ménages dont 17 Baka et 25 Konabembé et des relevés floristiques basés sur les données de présence-absence ont été effectués dans 161 exploitations agricoles. L'étude montre que l'insécurité alimentaire est une réalité chez les deux groupes ethniques. Elle est, cependant, plus marquée chez les Baka que chez les Konabembé. Les relevés floristiques ont permis de recenser un total de 170 espèces regroupées en 61 familles. Trente-quatre sont des plantes cultivées avec une présence marquée d'individus de la famille des Solanaceae ( 9 espèces) et 136 sont des espèces forestières sauvages accompagnant les cultures en champ, avec une grande richesse des individus de la famille des Sterculiaceae. Manihot esculenta est la culture la plus fréquente, tandis que Musanga cecropioides est l'espèce forestière la plus présente dans les exploitations. Au total 112 espèces dont 21 cultivées et 91 forestières forment le fonds floristique commun aux exploitations des Baka et des Konabembé. Cette communauté botanique masque tout de même quelques fines différences. Dix-neuf espèces sont présentes uniquement dans les exploitations Konabembé. Bien qu'il apparaisse très peu de différence en termes d'espèces cultivées entre les deux groupes ethniques, toutes espèces considérées, les exploitations des Baka sont floristiquement plus riches que celles des Konabembé.

Bien que ces écosystèmes cultivés renferment une diversité floristique importante, cette agrobiodiversité a du mal à répondre aux exigences d'une alimentation équilibrée. Des actions sont nécessaires à divers niveaux pour promouvoir des pratiques d'agriculture durable qui concilient conservation de la biodiversité, gestion durable des terres, sécurité alimentaire et résilience aux effets des changements climatiques.

Agrobiodiversity plays an important role as a source of income, a lever for the resilience of agricultural systems to climate change, a reservoir of nutrients, a basis for various ecosystem services such as pollination, soil fertility, and regulation of water cycles. Despite this potential, it is currently experiencing significant threat of erosion. This work characterizes agro-biodiversity in forest landscapes used by the Konabembe and the Baka ethnic groups in South-East Cameroon and the implications for household food security. Ethnobotanical surveys were conducted in 42 households, including 17 Baka and 25 Konabembé, and floristic surveys based on presenceabsence data were conducted to 161 farms. The study shows that food insecurity is a reality for both ethnic groups. It is, however, more marked among the Baka than the Konabembe. Floristic surveys allowed the identification of 170 species grouped in 61 families. Thirty four of them are cultivated crops mostly of Solanaceae family ( 9 espèces) and 136 are native forest species accompanying crops in farms, with a great richness of individuals of Sterculiaceae family. Manihot esculenta was the most frequent crop, while Musanga cecropioides was the native forest species most present in farms. Overall, 112 species (21 cultivated and 91 native forest species) form the common floristic pool of the Baka and Konabembé farms. This botanical community however masks some little differences. Nineteen species were present only in Konabembé farms. Although there is very little difference in terms of cultivated crops among the two ethnic groups, all species considered, Baka farms are floristically richer than those of Konabembé.

Although these cultivated ecosystems contain a significant floristic diversity, this agrobiodiversity is still far from meeting the requirements of a balanced diet. Action is needed at various levels to promote sustainable agriculture practices that harness biodiversity conservation, sustainable land management, food security and resilience to the effects of climate change. 
INDEX

Mots-clés : agrobiodiversité, Gribé, sécurité alimentaire, Cameroun

Keywords : agrobiodiversity, Cameroon, food security, Gribe

\section{AUTEURS}

\section{ÉVARISTE FONGNZOSSIE}

Université de Douala, École Normale Supérieure d'Enseignement Technique - BP 1872 Douala, Cameroun

\section{THOMAS MBANG NKONGO}

Université de Douala, Faculté des Sciences, Département de Biologie et Physiologie des Organismes Végétaux. BP 24157 Douala, Cameroun

\section{DIDIER DIBONG SIEGFRIED}

Université de Douala, Faculté des Sciences, Département de Biologie et Physiologie des Organismes Végétaux - BP 24157 Douala, Cameroun

\section{MARLENE NGANSOP}

Université de Yaoundé 1 - Faculté des Sciences, Département de Biologie et Physiologie des Organismes Végétaux. BP 8038 Yaoundé, Cameroun 